\title{
Reference Values of Platelet Aggregation in Impedance Aggregometry with Adenosine Diphosphoric Acid on Aggregometer Multiplate
}

\author{
DOI: 10.17691/stm2016.8.3.11
}

Received Apri1 24, 2015

O.V. Petrova, MD, PhD, Head of Clinical and Diagnostic Laboratory;

S.A. Shashin, MD, DSC, Cardiovascular Surgeon;

D.G. Tarasov, MD, PhD, Chief Doctor

Federal Center for Cardiovascular Surgery, Ministry of Health of the Russian Federation, 4 Pokrovskaya roshcha St.,

Astrakhan, 414011, Russian Federation

Current international standards recommend every laboratory to develop or confirm the existing in literature reference intervals for each laboratory value. We studied aggregation platelet function in 128 apparently healthy men and women of Astrakhan region (Russia) in clinical and diagnostic laboratory of Federal Center for Cardiovascular Surgery, Ministry of Health of the Russian Federation. The study involved impedance technique using aggregometer Multiplate (Verum Diagnostica, Germany), adenosine diphosphoric acid being used as an inductor. Age and gender had no significant effect on platelet aggregation values. Reference interval of platelet aggregation with adenosine diphosphoric acid on the aggregometer Multiplate in adult population of Astrakhan region was found to be 598.0-1,120.0 AU·min, where $\mathrm{AU} \cdot \mathrm{min}$ is the area under aggregation curve.

The stated platelet aggregation interval in adult population can be used as reference in Astrakhan region laboratories when working with similar analytical systems (automatic aggregometer Multiplate), since it was developed considering all peculiarities of developing reference groups and standardization of all laboratory research stages.

Key words: reference interval; platelet aggregation; inductor; adenosine diphosphoric acid; automatic aggregometer Multiplate.

Thrombocytes play an important role in clot formation in patients undergone cardiosurgical operations. Antithrombotic (antiaggregate) preparations are usually used to prevent and treat thrombosis [1].

Pharmaceutical preparations having various chemical structures and capable of inhibiting thrombocyte functions using different mechanisms are referred to antiaggregants. Application of antiaggregate agents (their dosage, results) must be controlled in patients having a risk of bleeding and thrombosis development by means of platelet aggregation function tests. For example, when clopidogrel (Plavix) and Prasugrel are used, the control is carried out by examining the platelet aggregation function in the presence of an activator adenosine diphosphoric acid [2-5].

Currently aggregometers are used in the laboratory practice to assess the platelet aggregation function [1-3]. The method of determining platelet aggregation is based on the ability of thrombocytes activated by inductors to form aggregates, which are analyzed by an aggregometer. Depending on the type of the material analyzed, the aggregometers are divided into analyzers working with whole blood, and analyzers which examine thrombocyte-enriched plasma. Aggregatometry may be impedance or optic according to the type of changes registration $[4,5]$.

In the recent years up-to-date impedance aggregometers have replaced the optic ones. They, in contrast to the optical devices, do not require special training of specialists in laboratory diagnosis and in preparation of the biological material, are simple in operation, reduce the time of blood testing.

A reference interval (RI) is used to assess the findings of laboratory examination. This index is a guide, reference information, which a physician needs for the correct interpretation of the laboratory investigation results. The upper and lower borders of RI serve as the lines of separation between health and disease in laboratory terms $[6,7]$.

Absence of regional Rl-indices of platelet function limits the use of modern impedance aggregometers in clinical practice. Besides, present-day Russian and international standards recommend each laboratory to establish RI for each laboratory parameter or to confirm data on RI existing in the literature [6-8].

The aim of the investigation was to establish reference intervals of platelet aggregation in the presence of adenosine diphosphoric acid using

For contacts: Olga V. Petrova, e-mail: students_asma@mail.ru 
Multiplate aggregometer for testing adult population of Astrakhan region.

Materials and Methods. For establishing $\mathrm{RI}$, a classic approach to its calculation using strict criteria of including and excluding from the investigation was used $[6,7]$.

To be a practically healthy person was a criterion for inclusion into the investigation.

People suffering from somatic pathology affecting homeostasis condition, or taking antithrombotic preparations were excluded from the study.

Investigations were carried out within the frames of prophylactic medical examination in the Federal Center for Cardiovascular Surgery (Astrakhan).

Reference group comprised 70 healthy men and 70 healthy women - people at the age of 40-60 years living in Astrakhan region.

The quality of laboratory investigation depends on preanalytic, analytic and postanalytic stages [9], therefore, all stages of our study have been standardized.

Standardization of preanalytic prelaboratory stage was provided by international and Russian standards, as well as local documents developed in the Federal Center for Cardiovascular Surgery (Astrakhan).

Blood specimens for investigation were collected by cubital vein puncture after the application of tourniquet (for about a minute) in a lying position with the help of two-component system for blood taking - disposable polypropylene tubes with 17 units/L of lithium-heparin (Sarstedt, Germany).

Blood specimens were delivered to the laboratory within 15-20 min after venipuncture and analyzed in 30$35 \mathrm{~min}$ after the delivery of the biological material to the clinical diagnostic laboratory.

Quality control of the biological material delivered to the laboratory was provided at the preanalytical laboratory stage in accordance with the international and domestic standards.

Standardization of the analytical stage was provided by:

annual technical maintenance of the automatic Multiplate aggregometer (Verum Diagnostica, Germany);

daily precision (correctness) testing of the work of the measuring items (electrodes) using electronic quality control in accordance with the operating instruction;

daily quality control of the impedance aggregometry using certified control materials for intralaboratory quality control in accordance with the operating instruction for the analyzer;

availability of the laboratory information system.

Aggregating platelet function was examined on a whole blood using impedance technique according to the manufacturer instruction by means of Multiplate aggregometer (Verum Diagnostica, Germany). Adenosine diphosphoric acid in $6.5 \mu \mathrm{M}$ concentration served as an inductor. The results of the platelet aggregation testing are presented as an area under the aggregation curve (AU.min).

All statistical procedures were performed with the help of the Statistica 6.0 for Windows program package (StatSoft, Inc., USA). The mean arithmetic X, standard deviation SD, median, the $5^{\text {th }}$ and $95^{\text {th }}$ percentiles of distribution were calculated. Univariate two-way analysis of variance was carried out, where gender and age were fixed factors; platelet aggregation in the presence of adenosine diphosphoric acid was a dependent variable. The type of distribution was determined using Kholmogorov-Smirnov criterion. Mann-Whitney (U) test was used to assess average differences between the groups. Divisions were considered statistically significant at $p<0.05$.

Statistical approaches recommended by the Federal standard GOST R 53022.3-2008 and standard A28-3 of the Clinical and Laboratory Standards Institute (CLSI) were used for RI determination [6, 7].

The investigation was performed in two steps. At the first step statistical outliers during the study of aggregation function of platelets with adenosine diphosphoric acid were defined and excluded from further work. The outliers were determined with the help of Tuky's method on the basis of "normal" value interval: $[\mathrm{Q} 1-1.5 \cdot \mathrm{IQR}, \mathrm{Q} 3+1.5 \cdot \mathrm{IQR}]$, where $\mathrm{Q} 1, \mathrm{Q} 3$ were the borders of the first and third quartiles, IQR=Q3-Q1 was interquartile range. The Tuky's method helped to exclude 12 results of platelet aggregation test that amounted to $11.7 \%$.

The second step was to determine the expediency of dividing the groups according to gender and age in the light of the obtained data about gender and age differences in platelet aggregation [10], when calculating RI by means of univariate two-way analysis of variance.

Results. The results of the univariate two-way analysis of variance of the expediency of dividing the groups according to gender and age when calculating $\mathrm{RI}$ of platelet aggregation in the presence of adenosine diphosphoric acid are presented in Tables 1 and 2 .

Statistically significant differences in platelet aggregation in the presence of adenosine diphosphoric acid (an average area under the curve) in men and women were not found (See Table 1).

Table 1

The results of the univariate two-way analysis of variance of the expediency of dividing the groups according to gender when calculating reference interval of platelet aggregation in the presence of adenosine diphosphoric acid

\begin{tabular}{lccc}
\hline \multicolumn{1}{c}{ Gender } & $\mathrm{n}$ & $\mathrm{X} \pm \mathrm{SD}(\mathrm{AU} \cdot \mathrm{min})$ & $\mathrm{p}$ \\
Men & 63 & $871.3 \pm 141.2$ & $>0.05$ \\
Women & 65 & $880.8 \pm 141.1$ & $>0.05$ \\
\hline
\end{tabular}


Table 2

The results of the univariate two-way analysis of variance of the expediency of dividing the groups according to age when calculating reference interval of platelet aggregation in the presence of adenosine diphosphoric acid

\begin{tabular}{lcc}
\hline \multirow{2}{*}{ Age (years) } & \multicolumn{2}{c}{ X \pm Men $($ AU.min) } \\
\cline { 2 - 3 } $40-50$ & $874.4 \pm 143.5(n=30)$ & Women \\
\hline $51-60$ & $880.9 \pm 138.9(n=33)$ & $890.1 \pm 150.5(n=30)$ \\
\hline
\end{tabular}

Statistically significant differences in platelet aggregation in the presence of adenosine diphosphoric acid in men and women depending on age were not revealed either (See Table 2).

Findings of the univariate two-way analysis of variance showed inexpediency of dividing the adult population of Astrakhan region according to gender and age when calculating RI of platelet aggregation.

In this connection, the data on the platelet aggregation test in men and women were combined in a single group in order to calculate mean value and standard deviation and to compare the obtained data with those indicated in the manufacturer instruction.

In Table 3 values of RI established by us for the adult population of Astrakhan region, and data from the manufacturer instruction are given.

The values of $5-95 \%$ for the established by us platelet aggregation RI did not practically differ from those indicated by the manufacturer in the instruction to the reagent kit.

There was no possibility to compare IR in the form of Xmean $\pm 1.96 S D$, since the manufacturer did not indicate it in the instruction to the kit.

Discussion. According to the known standards, method of Rl calculation depends on the size of the reference group and the type of the distribution of laboratory index values. If there are less than 120 people in the group and the distribution of laboratory indices is non-normal, IR is calculated in the form of $5-95 \%$, which means that normal laboratory indices are found in $90 \%$ of healthy persons and in $10 \%$ they are nonnormal. If the size of the group is over 120 people and the distribution of laboratory indices is normal, RI is calculated as Xmean \pm 1.96 SD, according to which $95 \%$ of healthy people are found to have normal laboratory indices, and $5 \%$ non-normal $[6,7]$.

The manufacturer in its instruction to the reagent kit indicated RI as 5-95\% and the number of people in the group equal to 150 (See Table 3), which may indicate non-normal distribution of platelet aggregation values.

In our investigation the distribution of platelet aggregation was normal and the group enrolled 128
Table 3

Values of the reference interval of platelet aggregation with adenosine diphosphoric acid

\begin{tabular}{lcc}
\hline \multicolumn{1}{|c}{ Results } & $\begin{array}{c}\text { Results obtained } \\
\text { by the authors } \\
(\mathrm{n}=128)\end{array}$ & $\begin{array}{c}\text { Data indicated } \\
\text { by the manufacturer } \\
{[11-13](\mathrm{n}=150)}\end{array}$ \\
\hline Xmean (AU·min) & 870.9 & - \\
\hline $\mathrm{SD}$ & 141.5 & - \\
\hline $\mathrm{Me}$ & 860.0 & 830.0 \\
\hline $5^{\text {th }}$ percentile & 620.0 & 548.0 \\
\hline $95^{\text {th }}$ percentile & $1,120.0$ & $1,167.0$ \\
\hline$\left.\mathrm{R}\right|^{*}[$ Me; 5; 95] (AU·min) & $620.0-1,120.0$ & $548.0-1,167.0$ \\
\hline $\mathrm{R}^{* *}$ (Xmean $\left.\pm 1.96 \mathrm{SD}\right)(\mathrm{AU} \cdot \mathrm{min})$ & $598.0-1,120.0$ & - \\
\hline
\end{tabular}

${ }^{*} \mathrm{RI}$ values obtained in accordance with the manufacturer instruction for the given aggregometer; ${ }^{*}$ RI values given in accordance with the recommendations of GOST R 53022.32008.

persons. According to A28-3 CLSI standard and GOST $\mathrm{R}$ 53022.3-2008, RI in case of normal distribution must be calculated by Xmean $\pm 1.96 \mathrm{SD}$ formula $[6,7]$. In this situation RI of platelet aggregation in the presence of adenosine diphosphoric acid is 598.0-1,120.0 AU-min.

Besides, gender and age do not significantly influence the values of platelet aggregation in the presence of adenosine diphosphoric acid. This differs from the data presented in the work [10], which revealed age and gender differences when establishing RI of platelet aggregation.

In conclusion the following should be noted once again. In recent years clinical diagnostic laboratories are actively being equipped by up-to-date analyzers. But absence of regional RI for laboratory indices makes their application difficult. If we use RI indicated in the instruction manual, it may lead to misinterpretation of the laboratory findings, since these RIs were established for different population.

In addition, in current domestic reference books on laboratory diagnostics there are almost no data about platelet aggregation RI that may be taken as a basis.

But at the same time, despite the RI indicated in the analyzer instruction manuals, the manufacturers of laboratory equipment recommend to establish RI of laboratory indices for the population of the region, where the given device is used.

In this connection we have carried out the procedure of establishing RI of platelet aggregation in the presence of adenosine diphosphoric acid using Multiplate aggregometer for the adult population of Astrakhan region. A reference interval of platelet aggregation with adenosine diphosphoric acid on the Multiplate aggregometer in healthy adult population of Astrakhan region was found to be 598.0-1,120.0 AU·min. 
Conclusion. The established platelet aggregation interval in adult population can be used as a reference one in Astrakhan region laboratories when working with similar analytical systems (automatic Multiplate aggregometer), since it was developed considering all peculiarities of forming reference groups and standardization of all laboratory research stages.

Study Funding and Conflicts of Interest. The work was done within the frames of the scientific research, conducted by the Federal Center of Cardiovascular Surgery of the Ministry of Health of the Russian Federation. The authors declare no conflicts of interest.

\section{References}

1. Barkagan Z.S., Momot A.P. Diagnostika $i$ kontroliruemaya terapiya narusheniy gemostaza [Diagnostics and controlled therapy of hemostasis]. Moscow; 2008; 292 p.

2. Charnaya M.A., Morozov Yu.A. Current antiplatelet drugs and their application in clinical practice (literature review). Kardiologiya i serdechno-sosudistaya khirurgiya 2009; 2(1): 34-40.

3. Siller J.M., Haberl K., Prillinger K., Panzer S., Lang I., Jilma B. The effect of antiplatet drugs clopidogrel and aspirin is less immediately after stent implantation. Thromb Res 2009; 123(6): 874-880, http://dx.doi.org/10.1016/j. thromres.2008.11.005.

4. Vavilova T.V. Gemostaziologiya v klinicheskoy praktike [Hemostasiology in clinical practice]. Saint Petersburg; 2005.

5. Dolgov V.V., Svirin V.P. Laboratornaya diagnostika narusheniy gemostaza [Laboratory diagnostics of hemostasis impairments]. Tver'; 2005; 277 p.

6. Kazakova M.S., Lugovskaya S.A., Dolgov V.V. The reference values of indicators of total blood analysis of adult working population. Klinicheskaya laboratornaya diagnostika 2012; 6: 43-49.

7. GOST $R$ 53022.3-2008. Tekhnologii laboratornye klinicheskie. Trebovaniya k kachestvu klinicheskikh laboratornykh issledovaniy. Chast' 3. Pravila otsenki informativnosti laboratornykh testov [GOST R 53022.3-2008. Clinical laboratory technologies. Requirements of quality of clinical laboratory tests. Part 3. Assessment of laboratory tests clinical significance]. 2009.

8. Clinical and Laboratory Standards Institute. EP28-A3C. Defining, establishing, and verifying reference intervals in the clinical laboratory, approved guideline — third edition. 2010.

9. Kishkun A.A. Rukovodstvo po laboratornym metodam diagnostiki [Manual for laboratory diagnostic techniques]. Moscow; 2007; 800 p.

10. Simonova O.I., Gordeeva O.B., NamazovaBaranova L.S., Botvin'eva V.V., Gorinova Yu.V., Soloshenko M.A. Sposob diagnostiki narusheniy agregatsii trombotsitov pri mukovistsidoze u detey. Patent RF №2533287 [Diagnostic technique of platelet aggregation impairment in cystic fibrosis in children]. Patent RF No.2533287. 2006.

11. Valarche V., Desconclois C., Boutekedjiret $T$., Dreyfus M., Proulle V. Multiplate whole blood impedance aggregometry: a new tool for von Willebrand disease. Thromb Haemost 2011; 9(8): 1645-1647, http://dx.doi.org/10.1111/ j.1538-7836.2011.04400.x.

12. Tóth O., Calatzis A., Penz S., Losonczy H., Siess W. Multiple electrode aggregometry: a new device to measure platelet aggregation in whole blood. Thromb Haemost 2006; 96(6): 781-788, http://dx.doi.org/10.1160/th06-05-0242.

13. Johnson A., Dovlatova N., Heptinstall S. Multiple electrode aggregometry and P2Y(12) antagonists. Thromb Haemost 2008; 99(6): 1127-1129, http://dx.doi.org/10.1160/ th08-01-0054. 